\title{
The Management of Cryptorchidism at the Pediatric Surgery Clinic of Tîrgu Mureș
}

\author{
Gozar H, Prișcă R, Derzsi Z \\ Pediatric Surgery Clinic, County Emergency Clinical Hospital, Tîrgu Mureș, Romania
}

\begin{abstract}
Introduction: Cryptorchidism is the most common abnormality of the male sexual development and one of the most common diagnoses in children hospitalized in the Pediatric Surgery Clinic. The diagnosis is mainly clinical: the testis cannot be seen or palpated in the scrotum. For this malformation, the treatment is always surgical: the orchiopexy.

Material and method: This is a retrospective study performed in 2012 in our clinic. The purpose of the study is to report our experience during a year in the management of this abnormality.

Results: There is a standard technique with two incisions (inguinal and scrotal) which was used in $77.12 \%$ of cases and a newer technique (Bianchi), with a single incision (scrotal), used in $22.88 \%$ of cases.

Conclusion: The results after surgery for cryptorchidism in every case were good, with both techniques.
\end{abstract}

Keywords: cryptorchidism, orchiopexy, testis

Received: 15 February 2013

\section{Introduction}

The term cryptorchidism describes a hidden or an obscure testis. This is the most common abnormality of the male sexual development [1].

Testicular development and descent depends on a complex interaction among genetic, endocrine, growth and mechanical factors [2]. Genetic causes are related to chromosomal alterations in the INSL3/ RXFP2 and AR genes [3]. As far as endocrine factors are concerned, androgens contribute to testicular descent. Leydig cells produce testosterone and stimulate the development of wolffian structures. Sertoli cells produce mullerian inhibiting factor (MIF), which causes the regression of the mullerian structures [4]. Epidermal growth factor stimulates the secretion of gonadotropin release hormone, involved also in the descent of the testis [4]. The genitofemural nerve may also aid in descent [1]. The role of the epididymis has been considered, because the gubernaculum inserts into it [4] and the mechanical role of traction and guide of the gubernaculum is well known. Birth weight and intra-abdominal pressure also appear to play a role in testicular descent [1].

The incidence of cryptorchidism at birth is about $2-9 \%$ $[2,5,6]$, being around $33 \%$ in premature infants [4].The majority of these retained testes descend spontaneously during the first few months, at the age of 1 year the incidence of cryptorchidism being 1-1.5\% [4,7]. After 1 year, the descent is unlikely to occur.

The undescended testis can be found in the inguinal region, perineum, femoral canal, penopubic area or in the

Correspondence to: Zoltán Derzs

E-mail: zoltan@netter.ro controlateral hemiscrotum. Cryptorchidism may also have a iatrogenic cause, when the testis is trapped in a scar or a retractile testis when the cremaster muscle retracts the testis into the inguinal canal. There are also intra-abdominal testes, vanishing or absent testes [4].

The most frequent anomalies associated with cryptorchidism are hernias, testicular maldevelopment, hypospadias, urinary tract abnormalities $[1,4]$.

The undescended testis is a malformation of the male genital organs and it may cause important problems. The purpose of this report is to bring attention on this malformation, highlighting the clinical aspects and the management of these cases.

\section{Material and method}

In this retrospective study we included all the 118 patients with cryptorchidism hospitalized in the Pediatric Surgery Clinic of Tîrgu Mureș between January $1^{\text {st }}$ and December $31^{\text {st }}, 2012$. Cryptorchidism was one of the most common diagnoses encountered in this period.

The diagnosis of undescended testis was mainly clinical: we could not see or palpate the testis in the scrotum. The patients were examined in a warm and relaxed environment. An important and useful determination was whether the testis was palpable or non-palpable. We made the palpation at the level of inguinal canal and then performed a "milking" down toward the scrotum. Sometimes we could find the testis and sometimes we could find an ectopic one (a testis placed out of the trace of the inguinal canal). We consider that a testis must be operated if it retracts in less than 10 seconds from the scrotum.

In monorchism or palpable testis, special laboratory investigations were not conducted. Patients with bilateral 
non-palpable testis or those with cryptorchidism associated with other malformations (especially hypospadias) underwent endocrine and genetic evaluation. Only in a few cases ultrasonography or MRI was recommended, but the latter required general anesthesia, being difficult especially in small children.

The treatment was always surgical, in the form of orchiopexy. There is a standard technique, which we used in most of the cases, and a newer one, an adaptation of the Bianchi operation. Our standard technique begins with an inguinal incision on the skin and then we open the external oblique aponeurosis. In the inguinal canal, we usually find the undescended testis. The gubernaculum is prepared and dissected. Sometimes there is a hernia sac which has to be separated from the cord structures and ligatured at the internal ring. If the cord is not long enough, a retroperitoneal dissection must be carried out. Then, the index is passed into the scrotum from the inguinal canal, in order to make a dartos pouch. From that pouch, a clamp is passed from the scrotum to the inguinal canal, grasping the vaginalis of the testis and taking the testis from the scrotal pouch. The orchiopexy is performed with fine sutures placed between the dartos and tunica vaginalis or tunica albugineea. The skin of the scrotum, then the inguinal structures are closed.

We also used an adaptation of the Bianchi operation, but only in the cases of palpable undescended testis. Using this technique we make a single transverse scrotal incision. A pouch is created at the level of the incision. The testis is dissected by the surrounding structures, being careful not to injure the cord. If there is a hernial sac, it is dissected from the cremaster and cord, as high as possible and is let to fall to the peritoneal cavity. The orchiopexy is made with two sutures between the dartos and tunica vaginalis. The scrotal skin is closed with two or three sutures.

The two procedures were performed under general anesthesia. The average operating time per an undescended testis was 20-30 minutes in a standard operation and 10-15 minutes in the adapted Bianchi operation. The orchiopexy may be carried out as one day surgery or may require one or two days of hospitalization in difficult cases. No antibiotics or antiinflamatory drugs were given. Follow-up was one week after the surgery.

\section{Results}

There were 118 boys in our study. Their age ranged between 1 and 14 years (mean age 5.79 years). The percentage of this operation was $6.86 \%$ from all the operations in our clinic. There were 50 undescended testes on the right side, 35 on the left side and 33 were bilateral.

We encountered 2 cases of ectopic testis (both in the infrainguinal region), 5 cases of testicular atrophy, 111 cases of undescended testis. Clinically, in 79 cases of cryptorchidism the testes could be palpated (66.94\%). As associated anomalies, we found 42 patients who also had inguinal hernia, 2 had hypospadias, 7 had phymosis and 5 had important obesity.
The standard procedure of orchiopexy was performed in 91 cases $(77.12 \%)$. In 27 cases $(22.88 \%)$ we used the adapted Bianchi technique.

The conversion of the adapted Bianchi technique to standard procedure was not necessary in any case. In all cases, the testes were positioned in a satisfactory manner into the scrotum and the cosmetic aspect was good. There were no surgical complications. The ectopic testis are usually smaller, so they could present hypotrophy.

\section{Discussion}

Cryptorchidism is an abnormality of the male genital organs, frequently encountered in Pediatric Surgery Units. The purpose of every physician, parent or child is to achieve a normally placed scrotal testis with a full potential for fertility and with no increase in the risk of testicular malignancy [8]. Monorchism or empty scrotum represents an important negative impact and leads to psychological problems in a boy's life. Therefore, it is important to perform the operation until the patient is not yet conscious about his sexual abnormality [2].

Standard orchiopexy requires two incisions: one in the inguinal region and the other at the scrotal level. The identification and dissection of the testis and cord are very important. A hernial sac is frequently encountered and this problem also has to be managed. The testis has to be placed in the scrotum, tension free. The adapted Bianchi technique requires only one incision, at the scrotal level. The operating time is shorter, the cosmetic aspect is improved and the toleration after surgery is better $[9,10]$.

The indication for every type of orchiopexy is different. The standard technique can be used for any type of cryptorchidism and the results are good. The Bianchi technique is recommended only for palpable testes. Fortunately, most of undescended testes are palpable at the external inguinal ring or even distally. The short distance between the external inguinal ring and the scrotum implies that in almost all cases it is possible to perform the orchiopexy with a minimally invasive operation, using a single scrotal incision. In these cases the results are also good [10].

In our statistic, the mean age for this operation was 5.79 years, which is high, given that the recommendation is to perform this kind of operation at the age of 1 or 2 years [2]. The problem is that parents take their children to a physician at a later age. Another interesting observation is that the majority of undescended testes were on the right side, as confirmed by other statistics [5].

We found that an important percentage of undescended testes were palpable (66.94\%). Other studies show similar figures [4]. These are the ideals cases: the operation — both the standard and the adpapted Bianchi technique - can be performed in easier conditions. The most important associated anomaly is inguinal hernia, similar to other statistics [1]. All the boys with testicular atrophy and those with hypospadias associated with cryptorchidism underwent endocrine evaluation. 
The adapted Bianchi operation was performed in 27 from the total of 118 cases. This is not a great number, but the results are good and our purpose is to increase the number of interventions using this technique. If we can not manage the situation through the transscrotal route, we can make an additional inguinal incision and convert the minimally invasive operation into a standard operation $[8,11]$.

The indication for orchiopexy is absolute. The risk of developing testicular cancer is 5 to 60 times greater for boys with cryptorchidism [4]. Also, their fertility may be affected. The alteration of testis in cryptorchidism is due to the effect of the higher local temperature [8]. Thus, the control of the scrotum during the first year of life is very important. Other indications for surgery are: a flat scrotum, orchiopexy can solve a possible testicular or Morgagni torsion or a hernia. The cosmetic aspect is also improved.

\section{Conclusions}

Cryptorchidism is the most frequent malformation in boys. The diagnosis is easy (empty scrotum) and a good evaluation must be done by a pediatric surgeon during the first year of life.

The treatment is important to be performed in time, before the age of 2 years. We admit that standard operation remains the golden technique, especially for non-palpable testes. Low transscrotal orchiopexy appears to be an excellent alternative to the standard inguinal orchiopexy for undescended testes located distally to the external inguinal ring, as we succeeded in many cases.

\section{References}

1. Elert A, Jahn K, Heidenreich $A$. The familial undescended testis. Klin Padiatr. 2003;215(1):40-5

2. Ritzen EM. Undescended testes: a consensus on management. Eur J Endocrinol. 2008;159(1):87-90. Bianchi A. The impalpable testis, Ann R Coll Surg Engl, 1995; 77: 3-6

3. Ferlin A, Zuccarello D, Zuccarello B: Genetic alterations associated with cryptorchidism. JAMA. 2008;300(19):2271-2276.

4. Keith LL, Shortliffe LD. Undescendeded testes and testicular tumors. In Pediatric Surgery by Aschraft KW, Murphy PJ, Patrick J, $4^{\text {th }}$ ed, Elsevier Saunders, Philadelphia, 2005:706-711.

5. Gapany C, Frey P, Cachat F. Management of cryptorchidism in children: guidelines, Swiss Med Wkly. 2008;138(33-34):492-498.

6. Ritzen EM, Kollin C. Management of undescended testes: how and when? Pediatr Endocrinol Rev. 2009;7(1):32-37.

7. Husmann DA. Testicular descent: a hypothesis and review of current controversis. Pediatr Endocrinol Rev. 2009;6(4):491-495.

8. Bianchi A. The impalpable testis. Ann R Coll Surg Engl. 1995;77:3-6.

9. Dayanc M, Kibar Y, Tahmaz L. Scrotal incision orchiopexy for undescended testis. Urology. 2004;64(6):1216-1218.

10. Handa R, Kale R, Harjai M. Single scrotal incision orchiopexy for palpable undescended testis. Asian J Surg. 2006;29(1):25-27.

11. Rajimwale A, Brant WO, Koyle MA. High scrotal (Bianchi) single-incision orchiopexy: a 'tailored' approach to the palpable undescended testis. Pediatr Surg Int. 2004;20(8):618-622. 